\title{
Lateral Wall of the Nose
}

National Cancer Institute

\section{Source}

National Cancer Institute. Lateral Wall of the Nose. NCI Thesaurus. Code C160988.

The outside wall of the nasal passages. 